OPEN ACCESS

Edited by:

Andrea Mari,

Careggi University Hospital, Italy

Reviewed by:

Carlo Terrone,

San Martino Hospital (IRCCS), Italy

Riccardo Campi,

Careggi Hospital, Italy

${ }^{*}$ Correspondence:

Yongguang Jiang

jyganzhen@163.com

Yong Luo

luoyonganzhen@163.com

${ }^{t}$ These authors have contributed equally to this work

Specialty section:

This article was submitted to Genitourinary Oncology,

a section of the journal

Frontiers in Oncology

Received: 09 March 2021 Accepted: 06 May 2021

Published: 03 June 2021

Citation:

Guo $P$, Wang $Y$, Han $Y$, Wei $D$, Zhao J, Li M, Jiang Y and Luo Y (2021) Oncological Outcomes of Patients With Different Pathological Features of pT3a Renal Tumor: A Systematic Review and Quantitative Synthesis.

Front. Oncol. 11:678459. doi: 10.3389/fonc.2021.678459

\section{Oncological Outcomes of Patients With Different Pathological Features of pT3a Renal Tumor: A Systematic Review and Quantitative Synthesis}

\author{
Pengju Guo ${ }^{\dagger}$, Yongxing Wang ${ }^{\dagger}$, Yili Han, Dechao Wei, Jiahui Zhao, Mingchuan Li, \\ Yongguang Jiang * and Yong Luo*
}

Department of Urology, Beijing Anzhen Hospital, Capital Medical University, Beijing, China

Purpose: To identify the differences in oncological outcomes for patients with different pT3a renal tumor invasion patterns and pathological features.

Methods: The protocol of this study was registered on PROSPERO (CRD42021234475). Relevant studies were identified by searching the PubMed, Cochrane library, Embase, and Web of Science databases. Cancer-specific survival (CSS) was selected as the endpoint. Pooled hazard ratio $(\mathrm{HR})$ and 95\% confidence interval $(\mathrm{Cl})$ extracted from multivariate Cox models were evaluated to identify the hazard association.

Results: A total of 22 studies, which enrolled 12384 patients were included for quantitative synthesis. Sinus fat invasion (SFI) + perinephric fat invasion (PFI) was associated with inferior CSS compared to SFI only $(p=0.02)$. Comparable CSS was observed between SFI and PFI $(p=0.57)$. SFI \pm PFI showed inferior CSS compared to PFI only $(p=0.0002)$. The presence of pelvicalyceal system invasion significantly increased the risk of cancer-specific mortality $(p=0.0005)$. Renal vein invasion $(R V I)$ indicated poor oncological outcomes in terms of CSS ( $p=0.002)$. The concomitant RVI and fat invasion (FI) significantly increased the risk of deterioration of CSS compared to RVI or $\mathrm{FI}$ ( $\mathrm{p}<$ 0.0001). Multiple invasion patterns translated into a significantly decreased CSS ( $p<$ 0.0001). Aggressive tumor behavior, including lymph node involvement $(p=0.006)$, distant metastases $(p<0.00001)$, sarcomatoid differentiation $(p<0.0001)$, necrosis $(p<$ $0.0001)$, Fuhrman grade III or IV ( $p<0.0001)$, positive margin $(p<0.0001)$, and tumor size $>7 \mathrm{~cm}(p<0.0001)$ were the predictors of inferior CSS. The lymphovascular invasion $(p=0.67)$ was indolent in terms of CSS.

Conclusion: This study confirmed the heterogenicity of pT3a renal tumors. Multiple invasion patterns could translate into a significantly decreased CSS, and SFI should not be merged in the SFI + PFI group. The presence of PSI or RVI could significantly increase the risk of cancerspecific mortality. Lymph node involvement, distant metastases, sarcomatoid differentiation, necrosis, high Fuhrman grade, positive margin, and size $>7 \mathrm{~cm}$ were the predictors of inferior CSS. A precise-risk grade of CSS for different invasion patterns including comprehensive combinations may be useful for the further refinements of the TNM system. 


\section{Systematic Review Registration: The current study was registered on PROSPERO, and the registration numbers is CRD42021234475.}

Keywords: renal tumor, pT3a, nephrectomy, cancer-specific survival, systematic review

\section{INTRODUCTION}

Since the publication of the sixth edition of the TNM staging system for renal tumors, the classification of T3a renal tumors has undergone several modifications. Although currently pT3a is defined as a tumor confined to the Gerota's fascia but exhibiting perinephric fat invasion (PFI), sinus fat invasion (SFI), renal vein invasion (RVI), or/and pelvicalyceal system invasion (PSI) regardless of tumor diameter, a realistic controversy is whether pT3a represents a heterogeneous histological group where different elements or a combination may indicate a significant difference in oncological prognosis. The EAU guidelines on renal cell cancer (RCC) state that tumors with SFI might be more aggressive than tumors with PFI, which was consistent with the findings of Thompson et al. $(1,2)$. However, several studies evaluating oncological outcomes for different pT3a renal tumor invasion patterns have failed to demonstrate the significant difference $(3,4)$. Lack of consensus on the outcomes of different extrarenal extension patterns may result from the unstandardized definitions for the histological assessment of fat invasion in the early years and discrepancies in study design $(2,5)$.

Our understanding of the heterogeneous behavior of renal tumors has been well advanced. The increasing interest in adjuvant treatment, immunotherapy and targeted therapies has prompted the need for more accurate staging of renal tumors (59). In the clinical context, some pT3a renal tumors are confirmed by postoperative pathology, and their incidence is usually underestimated. Therefore, it is important to accurately predict the prognosis of different $\mathrm{pT} 3 \mathrm{a}$ renal tumor invasion patterns to guide the follow-up protocols and evaluate the effect of postoperative therapies on survival. Given the continuing controversy over pT3a renal tumor staging, we undertook a systematic review and quantitative synthesis to determine whether pT3a represents a heterogeneous histological group and evaluate the oncological outcomes for different pT3a renal tumor invasion patterns and pathological features.

\section{METHODS}

This study was conducted according to the Preferred Reporting Items for Systematic Reviews and Meta-analysis (PRISMA) criteria, and the protocol was registered on PROSPERO for the study (CRD42021234475).

\section{Search Strategy}

We searched the PubMed, Cochrane Library, Embase, and Web of Science databases for studies investigating the oncological outcomes of $\mathrm{pT} 3 \mathrm{a}$ renal tumors from database inception to April
2021, using search terms integrated subject relevant terms ("renal tumor", "renal neoplasm," "renal cancer," and "renal cell carcinoma”) and staging terms (“T3a," “pT3a," “T3,” “pT3,” "renal vein invasion," and "urinary collecting system"). We also reviewed the references cited in the relevant articles to avoid omissions. The detailed Population, Intervention, Comparison, Outcome and Study design (PICOS) framework of the review was shown in Table 1. Only articles written in the English language were searched. All retrieved references were independently screened by two investigators (PG and YW) independently. When discordant decisions occurred, the senior authors (YJ and YL) were consulted to make final decisions.

\section{Study Selection, Data Extraction, and Quality Assessment}

The studies on the oncological outcomes for different pT3a renal tumor invasion patterns and pathological features following partial or radical nephrectomy (PN or RN) were included. Cancer-specific survival (CSS) was considered as the single endpoint of oncological outcomes. Conference abstracts, reviews, commentary, editorials, and letters were excluded but checked for cited references. The studies that did not provide CSS with a hazard ratio (HR) and corresponding $95 \%$ confidence interval (CI) or $\mathrm{p}$ value in the multivariate cox models were also excluded.

Two investigators independently extracted the data from each study. Extracted data included the name of the first author, year of publication, recruitment period, country or region, study type, sample size, surgery types, and size of pathological features of different pT3a renal tumor invasion patterns. HRs and 95\% CIs for CSS associated with different pT3a renal tumor invasion patterns and pathological features were extracted for quantitative synthesis. The quality of included studies was assessed using the Quality In Prognosis Studies (QUIPS) tool (10). The six bias domains when evaluating the literatures were study participation, study attrition, prognostic factor measurement,

TABLE 1 | The Population, Intervention, Comparison, Outcome and Study design (PICOS) framework of the review.

\begin{tabular}{|c|c|}
\hline Parameter & Inclusion criteria \\
\hline Population (P) & $\begin{array}{l}\text { Patients with pathological T3a and clinical or pathological } \\
\text { NO-1M0-1 renal tumor. }\end{array}$ \\
\hline Intervention (I) & Partial or radical nephrectomy \\
\hline Comparison (C) & $\begin{array}{l}\text { The cancer-specific survival of patients with different tumor } \\
\text { invasion patterns or pathological features. }\end{array}$ \\
\hline Outcome (O) & $\begin{array}{l}\text { Cancer-specific survival with the hazard ratio }(\mathrm{HR}) \text { and } \\
\text { corresponding } 95 \% \text { confidence interval }(\mathrm{Cl}) \text { or } \mathrm{p} \text { values in the } \\
\text { multivariate cox models. }\end{array}$ \\
\hline Study design (S) & $\begin{array}{l}\text { Randomized trials, population-based, single and multi-center } \\
\text { observational studies, and retrospective studies which were } \\
\text { published in English. }\end{array}$ \\
\hline
\end{tabular}


outcome measurement, study confounding, and statistical analysis and reporting. According to the items and considerations, the overall rating assessments were divided into low, moderate, and high risk of bias for each bias domain.

\section{Quantitative Synthesis and Analysis}

The comparisons of CSS between different tumor invasion patterns were evaluated by the pooled HRs with corresponding 95\% CIs. The statistical heterogeneity among studies was evaluated by the Cochrane $\mathrm{Q}$ test and quantified by $\mathrm{I}^{2}$ value. $\mathrm{I}^{2} \leq 50 \%$ indicated no or moderate heterogeneity, and a fixedeffect model was applied. On the contrary, $\mathrm{I}^{2}>50 \%$ indicated obvious heterogeneity, and a random-effect model was applied. The quantitative synthesis of HRs was evaluated by the inverse variance technique, and the quantitative synthesis of risk differences was evaluated by the Mantel-Haenszel test. The sensitivity analysis was conducted by one-removed analysis. Based on the recommendations of the Cochran manual, the evaluation of publication bias was conducted by using Egger's test only when there were 10 or more included studies (11). The certainty of the evidence were evaluated according to the Grading of Recommendations Assessment, Development, and Evaluation (GRADE) approach, which yields four levels of evidence (i.e., very low, low, moderate, and high) (12). All statistical tests were performed using Review Manager 5.4 (Cochrane Collaboration, Oxford, UK) and Stata 15.1 (StataCorp., College Station, Texas). Statistical significance was set at $\mathrm{p}<0.05$, and all specified $\mathrm{p}$ values were two-sided.

\section{RESULTS}

Among the 655 potential studies that were identified from the aforementioned databases, 139 studies were excluded due to duplication. After screening the titles and abstracts, 363 studies were found to be unsuitable for full text screening and were excluded. Based on the inclusion criteria, we conducted the fulltext evaluation of the remaining 153 studies, among which 23 studies did not focus on the patients with pT3a renal tumor, 16 studies did not report the HRs of the CSS, 52 studies did not compare the CSS for patients with different invasion patterns or pathological features, 13 studies were reviews, 10 study were comments, 13 studies were not published in English, and two studies were case reports. Among the 24 full-text articles assessed for eligibility, two studies that only reported the relevant HRs in the univariate Cox models were also excluded. Finally, 22 studies enrolling 12,384 patients were included in the quantitative synthesis (Figure 1) (2-7, 13-28).

The enrolled studies were published between 2005 and 2021, and included $12(54.5 \%)$ studies published over the past five years. All the enrolled studies were retrospective, and the recruited patients had no direct ipsilateral adrenal invasion. The recruited patients were from Asia, Europe, and USA. Ten studies focused only on patients following $\mathrm{RN}, 12$ studies included patients who received PN or RN (Table 2). Using the QUIPS tool, the risk of bias for each enrolled study was assessed and the results are shown in Figure 2. Also, two enrolled studies used data from the Surveillance, Epidemiology, and End Results

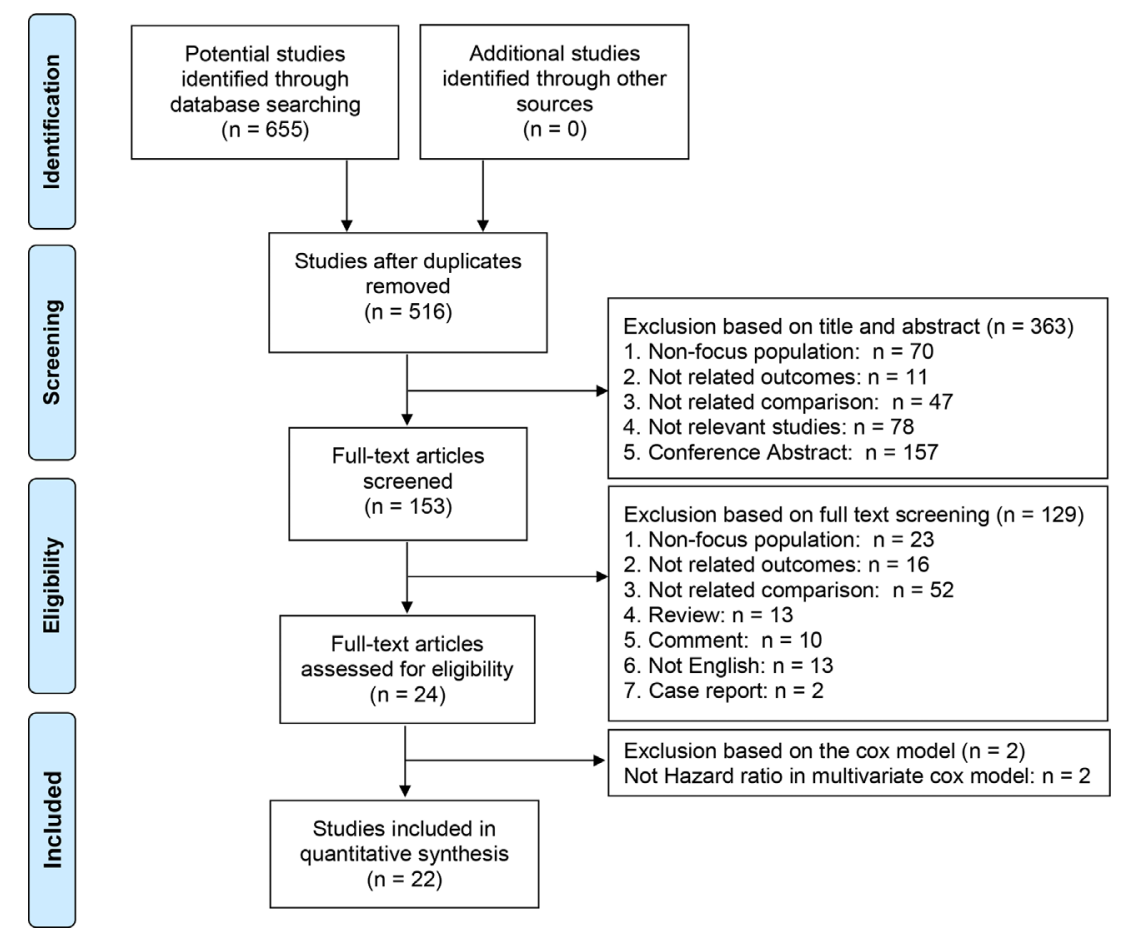

FIGURE 1 | Flow diagram of study selection. 
TABLE 2 | Characteristics of the included studies.

\begin{tabular}{|c|c|c|c|c|c|c|c|c|c|c|}
\hline Author & Year & Country & Recruitment period & Study design & Institution & Stage & Surgery type & Outcome & Cox model & Follow-up (mo) \\
\hline Thompson (2) & 2005 & USA & 1970-2002 & RTP & Single & No-1 MO-1 & $\mathrm{RN}$ & cSS & Multi & $72(24-408)^{\star}$ \\
\hline Margulis (13) & 2007 & USA & $1990-2006$ & RTP & Single & No-1 Mo-1 & $\mathrm{PN}, \mathrm{RN}$ & css & Uni, Multi & $33.5(6.1-158.6)^{\star}$ \\
\hline Poon (4) & 2009 & USA & 1988-2007 & RTP & Multiple & No-1 Mo-1 & PN, RN & CSS & Uni, Multi & $24(9-48)^{\star}$ \\
\hline Bedke (14) & 2009 & Germany & 1990-2007 & RTP & Single & No-1 MO-1 & $\mathrm{RN}$ & css & Uni, Multi & $34.8(14.4-109.2)^{*}$ \\
\hline Bertini (15) & 2009 & Italy & 1989-2006 & RTP & Single & No-1 MO-1 & RN & CSS & Uni, Multi & $38(2-240)^{\star}$ \\
\hline Kresowik (16) & 2010 & USA & $1997-2007$ & RTP & Single & No-1 Mo-1 & $\mathrm{PN}, \mathrm{RN}$ & css & Multi & $25.3(0-96.4)^{\star \star}$ \\
\hline Chen (24) & 2017 & China & 2006-2015 & RTP & Single & NO MO & $\mathrm{RN}$ & CSS & Uni, Multi & $31(3.4-109.7)$ \\
\hline Park (17) & 2017 & South Korea & $1997-2012$ & RTP & Single & NO MO & $\mathrm{PN}, \mathrm{RN}$ & css & Multi & $58.1(37.2-86.5)^{\star}$ \\
\hline Guo (18) & 2019 & China & 1979-2014 & RTP & Multiple & NO MO & $\mathrm{RN}$ & CSS & Uni, Multi & NA \\
\hline Shah (5) & 2019 & USA & 1970-2011 & RTP & Single & NO MO & $\mathrm{RN}$ & CSS & Uni, Multi & $111.6(81.6-160.8)^{\star}$ \\
\hline Wang (6) & 2020 & China & 2010-2016 & RTP & Multiple & No-1 MO-1 & $\mathrm{PN}, \mathrm{RN}$ & css & Multi & $24(10-46)^{\star}$ \\
\hline da Costa (19) & 2012 & Brazil & 1992-2009 & RTP & Single & No-1 Mo-1 & PN, RN & cSS & Multi & $28.6(3-60)^{\star \star}$ \\
\hline $\operatorname{Baccos}(20)$ & 2013 & Italy & 2000-2011 & RTP & Single & No-1 MO-1 & $\mathrm{RN}$ & css & Uni, Multi & $31(12-68.2)^{\star}$ \\
\hline Flood (21) & 2020 & Canada & $2011-2017$ & RTP & Single & No-1 Mo-1 & $\mathrm{RN}$ & CSS & Multi & $33.8(20.6-55.4)^{\star}$ \\
\hline Schiavina (22) & 2015 & Italy & 2000-2013 & RTP & Single & NO-1 MO & $\mathrm{RN}$ & css & Uni, Multi & $32(18-62)^{\star}$ \\
\hline Brookman-May (3) & 2015 & Germany & 1992-2010 & RTP & Multiple & NO MO & $\mathrm{PN}, \mathrm{RN}$ & css & Multi & NA \\
\hline Peng (23) & 2017 & China & 2007-2012 & RTP & Single & NO MO & $\mathrm{PN}, \mathrm{RN}$ & css & Multi & $35.5(10-86)^{\star}$ \\
\hline Oh (7) & 2018 & South Korea & 1988-2015 & RTP & Multiple & NO MO & $\mathrm{PN}, \mathrm{RN}$ & css & Uni, Multi & $38.8(\mathrm{NA})^{\star \star}$ \\
\hline Capitanio (25) & 2018 & Italy & 1988-2015 & RTP & Multiple & NO MO & $\mathrm{PN}, \mathrm{RN}$ & CSS & Multi & $52(N A)^{\star \star}$ \\
\hline Bailey (26) & 2017 & USA & $2001-2010$ & RTP & Single & No-1 MO-1 & $\mathrm{RN}$ & css & Uni, Multi & $98.4(72-129.6)^{\star}$ \\
\hline Garcia Marchinena (27) & 2019 & Argentina & 2000-2016 & RTP & Multiple & NO MO & $\mathrm{PN}, \mathrm{RN}$ & css & Uni, Multi & $21(1-194)^{\star}$ \\
\hline Lai (28) & 2021 & China & $2000-2018$ & RTP & Single & NO-x MO & $\mathrm{PN}, \mathrm{RN}$ & cSS & Uni, Multi & $48(\mathrm{NA})^{*}$ \\
\hline
\end{tabular}

\begin{tabular}{|c|c|c|c|c|c|c|c|c|c|c|c|}
\hline Author & Year & No.pT3a & No.PFI only (\%) & No.SFI only (\%) & No.SFI+PFI (\%) & No.SFI \pm PFI (\%) & No.FI (\%) & No.RVI \pm Fl (\%) & No.RVI+FI (\%) & No.RVI only (\%) & No.PSI (\%) \\
\hline Thompson (2) & 2005 & 205 & $162(79.0)$ & $16(7.8)$ & 27 (13.2) & $43(21)$ & NA & NA & NA & NA & NA \\
\hline Margulis (13) & 2007 & 365 & $199(54.5)$ & 96 (26.3) & 70 (19.2) & $166(45.5)$ & NA & NA & NA & NA & $331(90.7)$ \\
\hline Poon (4) & 2009 & 230 & 167 (72.6) & NA & NA & $63(27.4)$ & NA & NA & NA & NA & NA \\
\hline Bedke (14) & 2009 & 106 & $58(54.7)$ & 27 (25.5) & $21(19.8)$ & $48(45.3)$ & NA & NA & NA & NA & NA \\
\hline Bertini (15) & 2009 & 105 & $70(66.7)$ & $16(15.2)$ & $19(18.1)$ & 35 (33.3) & NA & NA & NA & NA & NA \\
\hline Kresowik (16) & 2010 & 110 & 36 (32.7) & $41(37.2)$ & $33(30)$ & 74 (67.3) & NA & NA & $57(51.2)$ & NA & NA \\
\hline Chen (24) & 2017 & 163 & $79(48.5)$ & $11(6.7)$ & NA & NA & NA & 87 (53.4) & NA & NA & $40(24.5)$ \\
\hline Park (17) & 2017 & 266 & $92(34.6)$ & $51(19.2)$ & 29 (10.9) & $80(30.1)$ & $172(64.7)$ & $94(35.3)$ & $69(25.9)$ & $25(9.4)$ & NA \\
\hline Guo (18) & 2019 & 1869 & 687 (36.8) & 381 (20.4) & $105(5.6)$ & $486(26.0)$ & $1173(62.8)$ & NA & $696(37.2)$ & NA & NA \\
\hline Shah (5) & 2019 & 563 & 114 (20.2) & $51(9.1)$ & NA & NA & NA & NA & NA & $163(29.0)$ & NA \\
\hline Wang (6) & 2020 & 5290 & 2569 (48.5) & 1975 (37.3) & $746(14.1)$ & NA & NA & NA & NA & NA & NA \\
\hline da Costa (19) & 2012 & 46 & NA & NA & NA & NA & $24(52.1)$ & NA & $11(23.9)$ & $11(23.9)$ & NA \\
\hline $\operatorname{Baccos}(20)$ & 2013 & 122 & NA & NA & NA & NA & 63 (51.6) & $59(48.4)$ & 41 (33.6) & $18(14.8)$ & NA \\
\hline Flood (21) & 2020 & 160 & NA & NA & NA & NA & NA & $97(61)$ & NA & NA & $24(15)$ \\
\hline Schiavina (22) & 2015 & 185 & NA & NA & NA & NA & NA & NA & NA & NA & NA \\
\hline Brookman-May (3) & 2015 & 1247 & NA & NA & NA & NA & 1036 (83.1) & $211(16.9)$ & NA & NA & NA \\
\hline Peng (23) & 2017 & 125 & NA & NA & NA & NA & 89 (71.2) & NA & NA & $36(28.8)$ & NA \\
\hline Oh (7) & 2018 & 211 & NA & NA & NA & NA & $124(58.8)$ & $87(41.2)$ & 47 (22.3) & $40(19.0)$ & NA \\
\hline Capitanio (25) & 2018 & 309 & 164 & 68 & NA & NA & NA & NA & NA & NA & NA \\
\hline Bailey (26) & 2017 & 325 & NA & NA & NA & NA & NA & NA & NA & NA & $27(8.3)$ \\
\hline Garcia Marchinena (27) & 2019 & 293 & $111(37.9)$ & $36(12.3)$ & 35 (11.9) & $118(40.3)$ & $253(86.3)$ & $91(31.1)$ & 57 (19.5) & $34(11.6)$ & 35 (11.9) \\
\hline Lai (28) & 2021 & 89 & NA & NA & NA & NA & $71(79.8)$ & $36(40.4)$ & $18(20.2)$ & $18(20.2)$ & NA \\
\hline
\end{tabular}

RTP, retrospective; PN, partial nephrectomy; RN, radical nephrectomy; NOS, Newcastle-Ottawa Scale; NA, not applicable; SFI, sinus fat invasion; PFI, perinephric fat invasion; RVI, renal vein invasion; FI, fat invasion; PSI, pelvicaliceal system invasion.

*Median (IQR).

**Mean (range). 


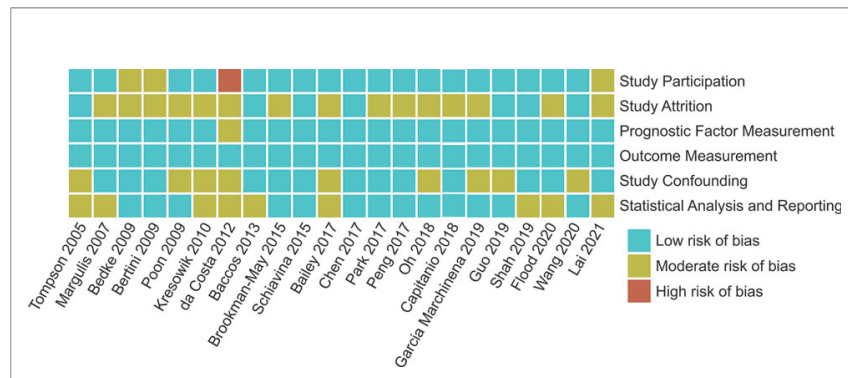

FIGURE 2 | Risk of Bias for each enrolled studies assessed by QUIPS (Quality In Prognosis Studies) tool.

(SEER) database $(6,18)$. However, due to the recruitment period and the fact that most reported pathological features were different, it was reasonable to include these two studies. When two studies reported the endpoints of the same pathological features, we selected the most recent outcomes, i.e., those reported by Wang et al., because of the enrolled patients identified between 2010 to 2016 (6).

\section{Cancer-Specific Survival of Different Invasion Patterns}

CSS results were available from 19 studies for different tumor invasion patterns. Although the pooled results revealed that SFI only $(\mathrm{n}=631)$ had comparable CSS to PFI only $(\mathrm{n}=903)(\mathrm{HR}$, 0.92; 95\% CI, 0.69-1.23; $\mathrm{p}=0.57 ; \mathrm{I}^{2}=20 \%$; Figure 3A), SFI + PFI $(\mathrm{n}=138)$ was associated with inferior CSS as compared to SFI only $(\mathrm{n}=422)\left(\mathrm{HR}, 1.97 ; 95 \% \mathrm{CI}, 1.13-3.42 ; \mathrm{p}=0.02 ; \mathrm{I}^{2}=2 \%\right.$; Figure 3B). SFI \pm PFI $(n=126)$ showed inferior CSS as compared to PFI only $(\mathrm{n}=290)$ (HR, 1.81; 95\% CI, 1.33-2.47; $\mathrm{p}=0.0002 ; \mathrm{I}^{2}=0 \%$; Figure $\left.3 \mathrm{C}\right)$. The pooled results revealed that patients with PSI $(n=102)$ had inferior CSS as compared to those without PSI $(\mathrm{n}=676)(\mathrm{HR}, 1.91 ; 95 \% \mathrm{CI}, 1.33-2.75 ; \mathrm{p}=$ $0.0005 ; \mathrm{I}^{2}=0 \%$; Figure 3D). Patients with RVI $(\mathrm{n}=531)$ had inferior CSS as compared to those without RVI $(\mathrm{n}=1484)(\mathrm{HR}$, 1.45; 95\% CI, 1.15-1.82; $\mathrm{p}=0.002 ; \mathrm{I}^{2}=47 \%$; Figure 3E), and the coexistence of RVI and fat invasion (FI) $(n=168)$ showed further deterioration of CSS as compared to RVI or FI ( $\mathrm{n}=477)$ (HR, 2.13; 95\% CI, 1.52-2.99; $\mathrm{p}=0.002 ; \mathrm{I}^{2}=0 \%$; Figure 3F). The multiple invasion pattern $(\mathrm{n}=1266)$ was associated with inferior CSS as compared to single pattern $(\mathrm{n}=1226)(\mathrm{HR}, 1.77 ; 95 \% \mathrm{CI}$, 1.49-2.09; $\mathrm{p}<0.00001 ; \mathrm{I}^{2}=0 \%$; Figure 3G). Using the GRADE approach, the certainty of SFI only vs. PFI only was low, while that of RVI+FI vs. RVI or FI was high. The certainty of SFI+PFI vs. SFI only, SFI \pm PFI vs. PFI only, PSI vs. non-PSI, RVI vs. nonRVI and multiple vs. single pattern were all moderate (Table 3).

\section{Cancer-Specific Survival of Different Pathological Features}

The pooled results revealed that lymph node involvement $(\mathrm{n}=$ 398; HR, 1.71; 95\% CI, 1.17-2.50; $\mathrm{p}=0.006 ; \mathrm{I}^{2}, 67 \%$; Figure 4A), distant metastases ( $\mathrm{n}=726$; HR, 3.36; 95\% CI, 2.88-3.91; $\mathrm{p}<$ 0.00001; $\mathrm{I}^{2}=42 \%$; Figure 4B), sarcomatoid differentiation $(\mathrm{n}=$ 436; HR, 2.09; 95\% CI, 1.78-2.46; $\mathrm{p}<0.00001 ; \mathrm{I}^{2}=12 \%$; Figure
4C), Fuhrman grade III or IV ( $\mathrm{n}=1737$; HR, 2.70; 95\% CI, 2.18 3.34; $\mathrm{p}<0.00001 ; \mathrm{I}^{2}=0 \%$; Figure 4D), necrosis $(\mathrm{n}=640 ; \mathrm{HR}$, 1.96; 95\% CI, 1.54-2.49; $\mathrm{p}<0.00001 ; \mathrm{I}^{2}=0 \%$; Figure 4E), tumor size $>7 \mathrm{~cm}(\mathrm{n}=1571$; HR, 1.77; 95\% CI, 1.46-2.15; $\mathrm{p}<0.00001$; $\mathrm{I}^{2}=1 \%$; Figure $\left.4 \mathbf{F}\right)$, and positive margin $(\mathrm{n}=28 ; \mathrm{HR}, 7.61 ; 95 \%$ CI, 4.12-14.04; $\mathrm{p}<0.00001 ; \mathrm{I}^{2}=32 \%$; Figure 4G) were associated with inferior CSS. The lymphovascular invasion (LVI) ( $\mathrm{n}=159$; $\mathrm{HR}, 1.11$; 95\% CI, 0.69-1.80; $\mathrm{p}=0.67 ; \mathrm{I}^{2}=0 \%$; Figure 4H) was not a predictor of inferior CSS. Using the GRADE approach, the certainty of lymph node involvement and LVI were low. Tumor size $>7 \mathrm{~cm}$ and necrosis showed moderate certainty. The certainty of metastases, sarcomatoid differentiation, Fuhrman grade III or IV, and positive margin status were all high.

\section{Sensitivity Analysis}

The sequential omission of a single study was conducted to test the stability of pooled results. The merged HRs for CSS did not significantly change, which revealed the robustness of the results. (Figure 5).

\section{Publication Bias}

The evaluation of publication bias was conducted using Egger's test only when there were 10 or more included studies. There was no significant publication bias in CSS study of sarcomatoid differentiation (Egger's test $\mathrm{p}=0.232$ ).

\section{DISCUSSION}

Classifying tumors from the surgical perspective and optimizing prognostic discrimination are the cardinal principles in the refinements of the TNM system as T3a renal tumor contains a wide range of four patterns of extrarenal extension regardless of tumor diameter and is confirmed by postoperative pathology in general. Nevertheless, the accuracy and rationality of T3a classification have been questioned in the context of inconsistency of individual oncological outcomes reported in the last fifteen years $(3,16,18,21)$. In the current study, we integrated the available clinical evidence and experience by conducting this systematic review and quantitative synthesis.

The major findings of the current study are the following: First, the moderate-certainty evidence suggests that SFI + PFI was associated with inferior CSS as compared to SFI only. The low-certainty evidence of comparable CSS between SFI only and PFI only and the moderate-certainty evidence of inferior CSS of SFI \pm PFI compared to PFI only further support the above findings. Several studies, which merged SFI only and SFI + PFI into a single group given the oncological equipoise derived from their cohorts, may be imprecise $(2,15)$. Second, moderatecertainty evidence suggests that the presence of PSI indicated significantly poor oncological outcome, with a 1.91 times increased risk of cancer-specific mortality (CSM). Although numerous studies have highlighted the adverse effect of PSI on oncological outcomes, the independent prognostic value of PSI has been excluded from the second to seventh edition of AJCC 


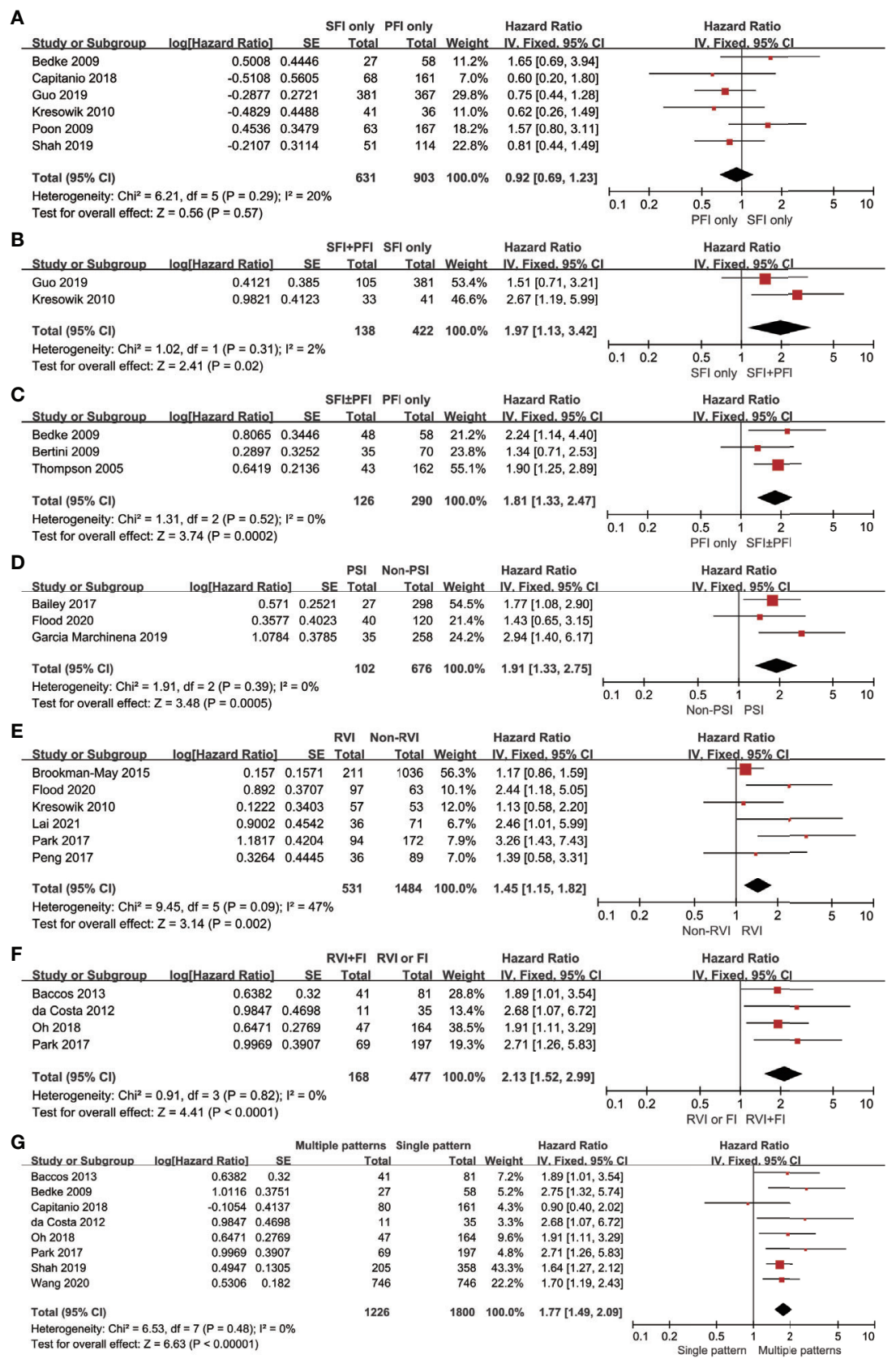

FIGURE 3 | Forrest plots of hazards ratio (HR) evaluating the significant predictors of cancer-specific survival for different pT3a renal tumor invasion patterns. (A): SFI only vs PFI only; (B): SFI + PFI vs SFI only; (C): SFI \pm PFI vs PFI only; (D): PSI vs non-PSI; (E): RVI vs non-RVI; (F): RVI + FI vs RVI or Fl; (G) Multiple patterns vs Single pattern. SFI, sinus fat invasion; PFI< perinephric fat invasion; PSI, pelvicaliceal system invasion; RVI, renal vein invasion; Fl, fat invasion.

TNM system $(26,27,29-31)$. Palapattu et al. reported a strong relationship between PSI, lymph node invasion and distant metastases (32). Third, high-certainty evidence suggests that the concomitance of RVI and FI significantly increased the risk of deterioration of CSS as compared to RVI or FI supported the finding that multiple invasion patterns translated into moderatecertainty evidence of significantly decreased CSS. However, most contemporary studies that reported the prognostic heterogeneity of T3a RCC failed to comprehensively explore the survival difference among the various combinations. A precise-risk grade of CSS for different invasion patterns, including comprehensive combinations, may be useful for further refinements of the TNM system. Finally, high-certainty evidence indicates that distant metastases, sarcomatoid differentiation, high Fuhrman grade and positive margin were the predictors of inferior CSS. Tumor size $>7 \mathrm{~cm}$ and necrosis 
TABLE 3 | The overall quality of evidence according to the GRADE (Grading of Recommendations Assessment, Development, and Evaluation) approach.

\begin{tabular}{|c|c|c|c|c|c|c|c|c|c|c|c|c|}
\hline \multirow[t]{2}{*}{ Comparison } & \multicolumn{6}{|c|}{ Certainty assessment } & \multicolumn{2}{|c|}{ No. of patients } & \multicolumn{2}{|c|}{ Effect } & \multirow[t]{2}{*}{ Certainty } & \multirow[t]{2}{*}{ Importance } \\
\hline & No. of Studies & Risk of bias & Inconsistency & Indirectness & Imprecision & Large effect & Case & Control & HRs & $95 \% \mathrm{Cl}$ & & \\
\hline SFI only vs PFI only & 6 & not serious & not serious & not serious & serious & No & 631 & 903 & 0.92 & $0.69-1.23$ & $\oplus \oplus \bigcirc \bigcirc$ LOW & IMPORTANT \\
\hline SFI+PFI vs SFI only & 2 & not serious & not serious & not serious & not serious & No & 138 & 422 & 1.97 & $1.13-3.42$ & $\oplus \oplus \oplus \bigcirc$ MODERATE & IMPORTANT \\
\hline $\mathrm{SFI} \pm \mathrm{PFI}$ vs PFI only & 3 & not serious & not serious & not serious & not serious & No & 126 & 290 & 1.81 & $1.33-2.47$ & $\oplus \oplus \oplus \bigcirc$ MODERATE & IMPORTANT \\
\hline PSI vs non-PSI & 3 & not serious & not serious & not serious & not serious & No & 102 & 676 & 1.91 & $1.33-2.75$ & $\oplus \oplus \oplus \bigcirc$ MODERATE & IMPORTANT \\
\hline RVI vs non-RVI & 6 & not serious & not serious & not serious & not serious & No & 531 & 1484 & 1.45 & $1.15-1.82$ & $\bigoplus \oplus \oplus \bigcirc$ MODERATE & IMPORTANT \\
\hline RVI+FI vs RVI or FI & 4 & not serious & not serious & not serious & not serious & Yes & 168 & 477 & 2.13 & $1.52-2.99$ & $\oplus \oplus \oplus \oplus \mathrm{HIGH}$ & IMPORTANT \\
\hline Multiple vs Single pattern & 8 & not serious & not serious & not serious & not serious & No & 1226 & 1800 & 1.77 & $1.49-2.09$ & $\bigoplus \oplus \oplus \bigcirc$ MODERATE & IMPORTANT \\
\hline $\begin{array}{l}\text { Node } \\
\text { N1 vs NO/x }\end{array}$ & 6 & not serious & serious & not serious & not serious & No & 398 & 1912 & 1.71 & $1.17-2.5$ & $\oplus \oplus \bigcirc \bigcirc$ LOW & IMPORTANT \\
\hline $\begin{array}{l}\text { Metastases } \\
\text { M1 vs M0 }\end{array}$ & 8 & not serious & not serious & not serious & not serious & Yes & 726 & 1844 & 3.36 & $2.88-3.91$ & $\oplus \oplus \oplus \oplus \mathrm{HIGH}$ & IMPORTANT \\
\hline $\begin{array}{l}\text { Sarcomatoid } \\
\text { Yes vs No }\end{array}$ & 10 & not serious & not serious & not serious & not serious & Yes & 436 & 3185 & 2.09 & $1.78-2.46$ & $\bigoplus \oplus \oplus \oplus \mathrm{HIGH}$ & IMPORTANT \\
\hline $\begin{array}{l}\text { Fuhrman grade } \\
\text { III or IV vs II or I }\end{array}$ & 8 & not serious & not serious & not serious & not serious & Yes & 1737 & 1065 & 2.7 & $2.18-3.34$ & $\bigoplus \oplus \oplus \oplus \mathrm{HIGH}$ & IMPORTANT \\
\hline $\begin{array}{l}\text { Necrosis } \\
\text { Yes vs No }\end{array}$ & 6 & not serious & not serious & not serious & not serious & No & 640 & 699 & 1.96 & $1.54-2.49$ & $\bigoplus \bigoplus \bigoplus \bigcirc$ MODERATE & IMPORTANT \\
\hline $\begin{array}{l}\text { Size } \\
>7 \mathrm{~cm} \text { vs } \leq 7 \mathrm{~cm}\end{array}$ & 5 & not serious & not serious & not serious & not serious & No & 1571 & 1811 & 1.77 & $1.46-2.15$ & $\bigoplus \bigoplus \oplus \bigcirc$ MODERATE & IMPORTANT \\
\hline $\begin{array}{l}\text { Margin status } \\
\text { positive vs negative }\end{array}$ & 6 & not serious & not serious & not serious & not serious & Yes & 28 & 1154 & 7.61 & $4.12-14.04$ & $\oplus \oplus \oplus \oplus \mathrm{HIGH}$ & IMPORTANT \\
\hline LVI vs non-LVI & 3 & not serious & not serious & not serious & not serious & No & 159 & 290 & 1.11 & $0.69-1.8$ & $\oplus \oplus \bigcirc \bigcirc$ LOW & IMPORTANT \\
\hline
\end{tabular}




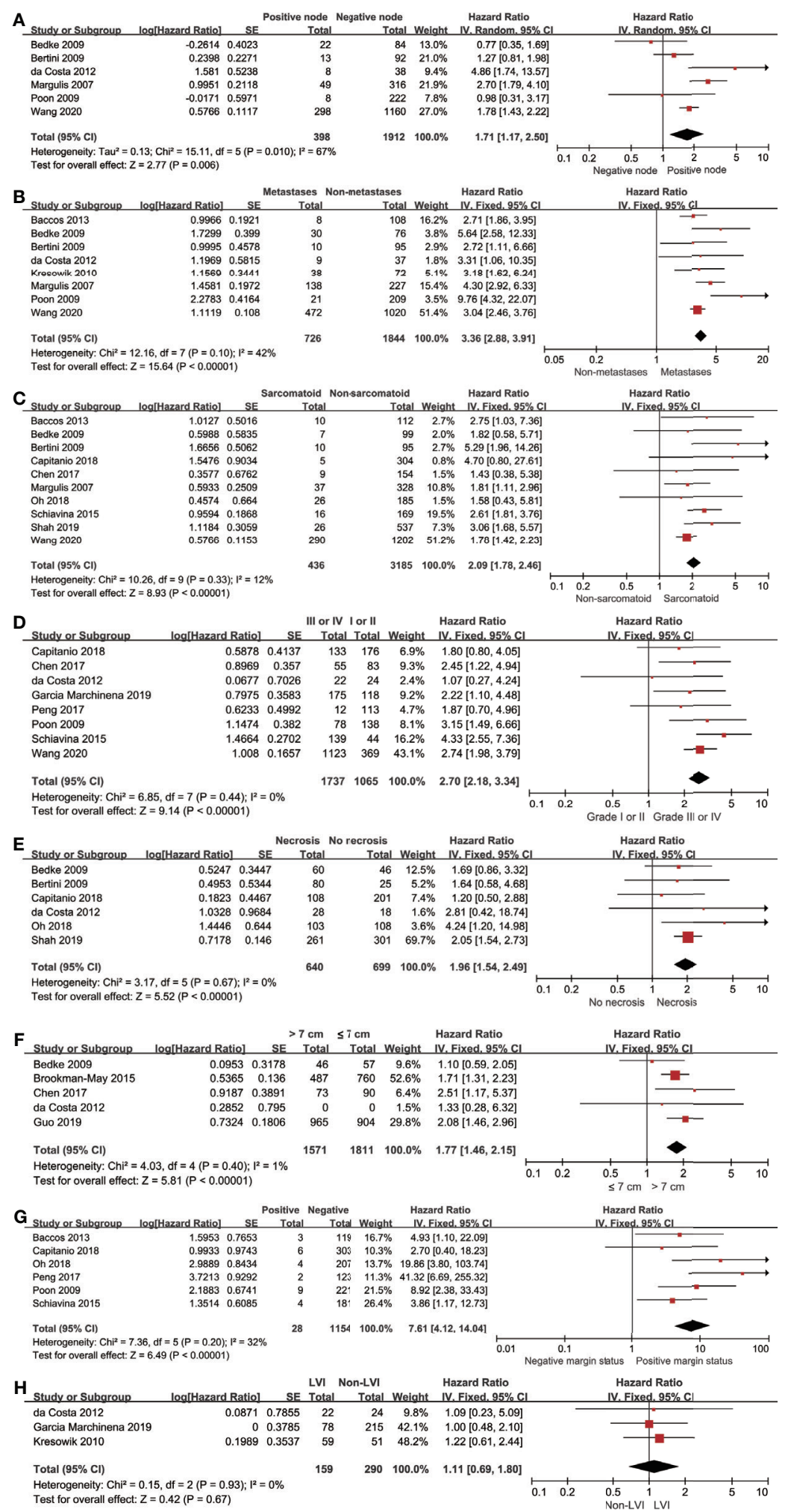

FIGURE 4 | Forrest plots of Hazards ratio (HR) evaluating the significant predictors of cancer-specific survival for different pathological features of pT3a. (A): lymph node involvement; (B): distant metastases; (C): sarcomatoid differentiation; (D): Fuhrman grade (III, IV vs I, II); (E): tumor necrosis; (F): tumor size (>7 cm vs $\leq 7 \mathrm{~cm}$ ); (G): positive margin status; (H): lymphovascular invasion. 
A

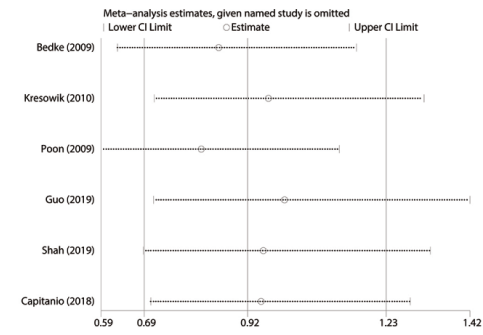

c
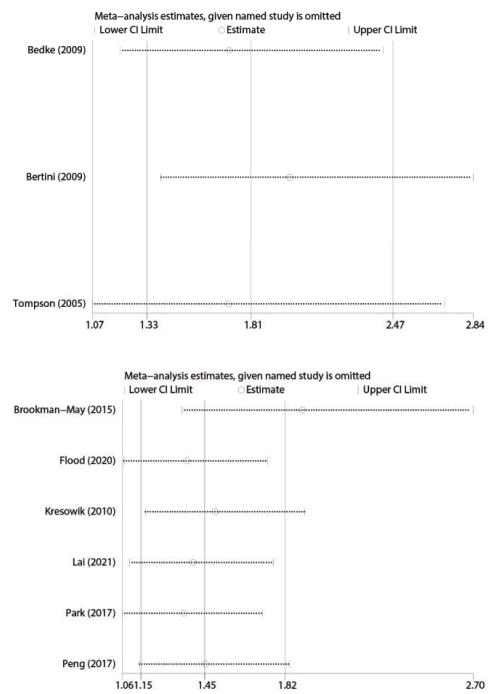

G

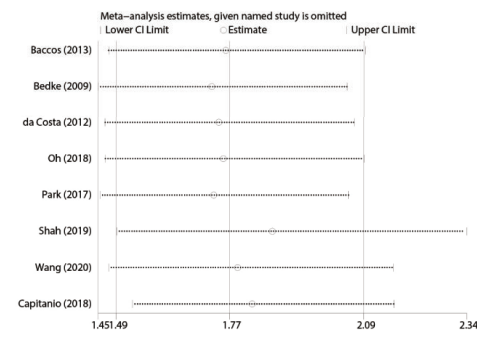

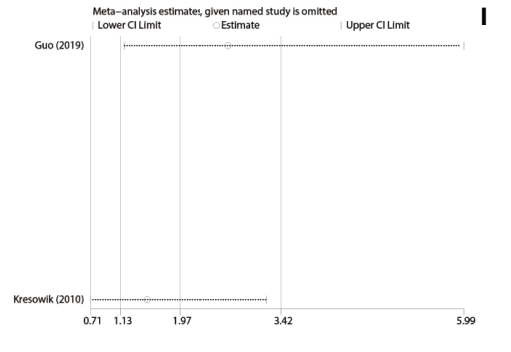

D
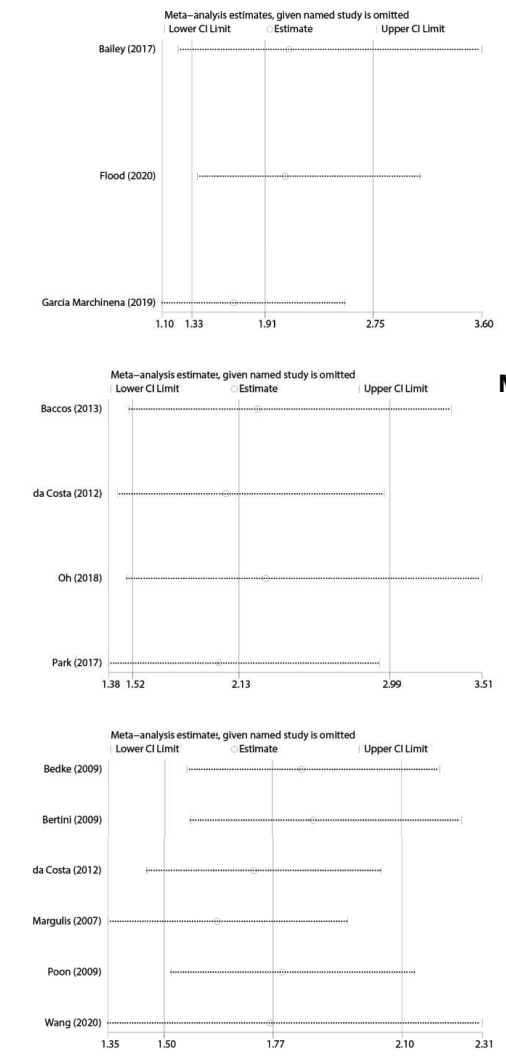

K

M

\section{I}
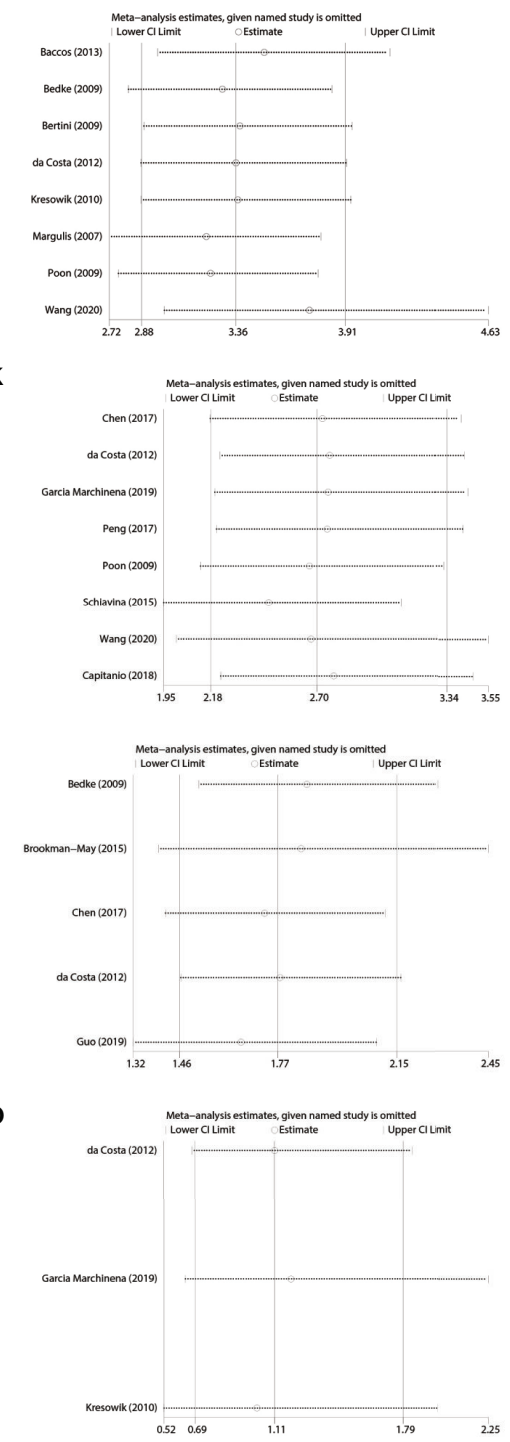

J

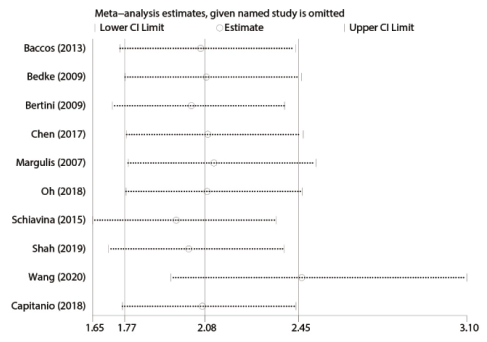

L

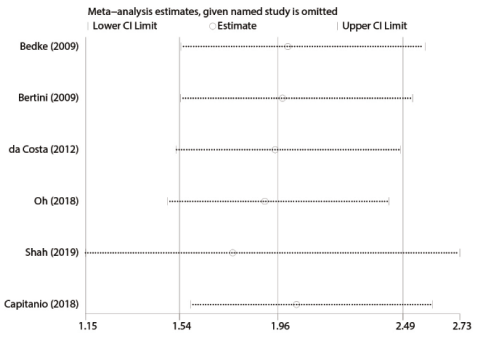

N

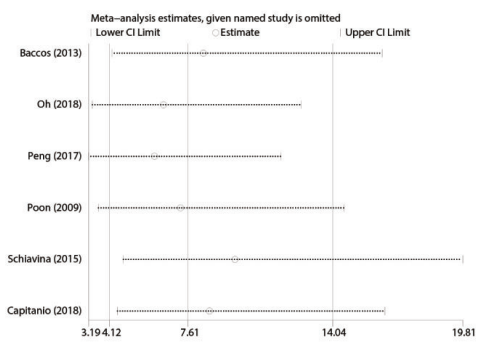

FIGURE 5 | Sensitive analysis of the included studies by one-removed analysis. (A): SFI only vs PFI only; (B): PFI + SFI vs SFI only; (C): SFI \pm PFI vs PFI only; (D): PSI vs non-PSI; (E): RVI vs non RVI; (F): RVI + FI vs RVI or FI; (G): multiple vs single pattern; (H): Iymph node involvement; (I): distant metastases; (J): sarcomatoid differentiation; (K): Fuhrman grade (III, IV vs I, III); (L), tumor necrosis; (M): tumor size (> 7 cm vs $\leq 7$ cm); (N), positive margin status; (O), lymphovascular invasion; SFI, sinus fat invasion; PFI, perinephric fat invasion; PSI, pelvicaliceal system invasion; RVI, renal vein invasion; Fl, fat invasion. 
also increased the risk of deterioration of CSS, which represents the moderate-certainty evidence. The low-certainty evidence suggests that lymph node involvement might increase the risk of CSM and the lymphovascular invasion was indolent in terms of CSS. The comparable CSS between SFI only and PFI only and the indolent impact of lymphovascular invasion on the survival are inconsistent with the EAU guidelines on RCC, which underlines the prognostic value of several anatomical and histological factors (1). This may require further validation due to the low-certainty evidence.

The inevitable risk of bias caused by the type of surgery that might affect the results of the included studies should be highlighted, even though it had been adjusted in the studies, which included patients undergoing PN or RN. Several studies reported comparable CSS for upstaged $\mathrm{pT} 3 \mathrm{a}$ PN patients compared to $\mathrm{pT} 3 \mathrm{a}$ RN patients $(33,34)$. However, the significantly smaller tumor size of the PN cohort compared to the tumor size of the $\mathrm{RN}$ cohort indicated that organ confined tumors are susceptible to receive PN. Given the absence of the standardized pathological protocol of capsular invasion in the early years, the classification of renal capsular invasion patterns was an unreliable prognostic variable in some previous studies $(2,35)$. The recommended routine histopathological examination of perirenal fat was conducted on a discounted basis among patients with peripheral renal tumor since the specimens of renal sinus fat were not systematically collected during PN, especially in the context of the PN enthusiasm (36). The aforementioned factors might have led to the underreported frequency of SFI. Grignon et al. noted that pT1b and pT2 renal tumors probably represented a shrinking proportion when the renal sinus was carefully evaluated (37). In the last two decades, TNM staging classification system for renal tumors was refined three times, which may affect the accuracy and manifolds of pT3a and the heterogeneity of study designs despite minor changes.

Given the increasing PN implementation, the realistic concern is the positive margin, which occurs more frequently in patients with aggressive features, including pT2a, pT3a, and grade III-IV $(38,39)$. Shah et al. reported that positive margin significantly increased the rate of recurrence, especially among patients with aggressive pathological features, including pT2T3a, high Fuhrman grade, and clear cell histology (39). In their study, recurrence was observed in almost one third of patients who were up staged to pT3a after PN (40). Bensalah et al. found that positive margin did not cause a decrease CSS; however, the fact that the mean tumor size was $3.5 \pm 2 \mathrm{~cm}$, and almost $90 \%$ of the positive margin cohort were patients with T1-2 RCC made their conclusions not necessarily applicable to patients with pT3a patients (41). Although several studies mentioned the controversial impact of positive margin on the oncological prognosis among patients with localized RCC, according to the current results that identified more than seven times risk of CSM in patients with pT3a RCC and postoperative positive margin compared to those with negative margin, the weak recommendation of EAU guidelines for intensive follow-up of patients with positive margin may be imprecise $(1,42,43)$.

Several studies have highlighted the impact of tumor size on CSS for T3a renal tumor $(3,18,22,39,44-48)$. In light of the agreement of some studies in which a cutoff of $7 \mathrm{~cm}$ was recommended as a prognosis prediction for $\mathrm{T} 3 \mathrm{a}$ renal tumor and the applicability of the refinement for the current TNM classification, only the results that considered tumor diameter as a binary variable by using a cutoff of $7 \mathrm{~cm}$ were merged in the current study. We found that patients with pT3a renal tumor $>$ $7 \mathrm{~cm}$ experienced an additional $77 \%$ risk of CSM, which was consistent with the findings of Brookman-May et al, reporting that tumor size was identified with the highest prediction accuracy by increasing $71 \%$ risk of CSM with a $7 \mathrm{~cm}$ cutoff (3). Although the tumor size did not result as a predictor of prognosis in several studies, which cannot be ignored, this is not necessarily contrary to our results and should be further analyzed in the context of the design of the studies and evaluation of patients $(14,19)$. Whether the impact of tumor size on prognosis can induce T3a and T1/T2 reintegration needs further validation. Chevinsky et al. reported that pT3a had significantly inferior RFS compared to pT1/T2 (45). Chen et al. found that patients with pT3a renal cell carcinoma showed poorer disease-free survival (DSS) as compared to pT1a, pT1b, pT2a, and pT2b. However, Yoo et al. reported a comparable CSS and DSS between pT2 and pT3a $\leq 7 \mathrm{~cm}$ (49).

The results of the current study may be used to guide the follow-up protocols and select patients suitable for adjuvant therapy after nephrectomy. A compact interval of surveillance may be vital for patients with aggressive factors. Although limited evidence suggested that compact postoperative imaging intervals did not result in the early detection of recurrence, which would benefit survival, the EAU guidelines on RCC recommend a risk-based approach to stratify follow-up for different patients, based on the individual aggressive anatomical, histological and clinical factors $(1,50)$. The S-TRAC trail exhibited superior disease-free survival (DFS) with sunitinib support. The PROTECT study also reported an improved DFS in the intention to treat pazopanib $800 \mathrm{mg}$ population (51). Among the highest-risk subpopulation, the ATLAS study found that axitinib translated into a $36 \%$ and $27 \%$ reduction in risk of a DFS event per investigator and by independent review committee, respectively (52). However, the recent SORCE trial results, which focused on the DFS and overall survival (OS) in patients with an intermediate or high risk of recurrence, failed to offer positive evidence of sorafenib (53). A recent meta-analysis revealed that adjuvant use of tyrosine kinase inhibitors (TKI) did not translate into improved OS, but showed a benefit in DFS in overall and high-risk populations (54). Due to the lack of sufficient evidence that adjuvant therapy with vascular endothelial growth factor receptor (VEGFR) -TKI offers survival benefits for patients with high-risk RCC, the EAU guidelines on RCC do not recommend the adjuvant therapy after nephrectomy (1). However, heterogeneity among the enrolled patients could be the main cause of the negative results (55). In light of the non-strict inclusion criteria of previous studies, supplemental randomized trials are necessary to determine whether patients with aggressive patterns or characteristics of pT3a renal tumors may benefit from adjuvant treatment.

The present study has some limitations. First, the retrospective nature of the included studies inevitably led the selection bias. 
Second, the inevitable risk of bias caused by the type of surgery might affect the results. Third, most contemporary studies failed to comprehensively explore the survival difference among the various combinations. Fourth, the determination of the patterns of pT3a renal tumor invasion and pathological features were made by different pathologists, probably based on different criteria. Finally, a small sample of some included studies increased the variability of results.

\section{CONCLUSION}

The current study identified the heterogenicity of pT3a renal tumors. Multiple invasion patterns could translate into a significantly decreased CSS, and SFI only should not be merged with the SFI + PFI group. The presence of PSI or RVI could significantly increase the risk of cancer-specific mortality. Lymph node involvement, distant metastases, sarcomatoid differentiation, necrosis, high Fuhrman grade, positive margin, and tumor size $>7 \mathrm{~cm}$ are the predictors of inferior CSS. The follow-up protocols and postoperative therapies after nephrectomy should be conducted with individuation according to a risk-based approach for stratification based on these aggressive pathological characteristics. External validation and a precise-risk grade of CSS for different invasion patterns,

\section{REFERENCES}

1. Ljungberg B, Albiges L, Bedke J, Bex A, Capitanio U, Giles RH, et al. (2021). Eau Guidelines on Renal Cell Carcinoma. In: Presented at the EAU Annual Congress Milan 2021. Arnhem, The Netherlands: EAU Guidelines Office

2. Thompson RH, Leibovich BC, Cheville JC, Webster WS, Lohse CM, Kwon ED, et al. Is Renal Sinus Fat Invasion the Same as Perinephric Fat Invasion for pT3a Renal Cell Carcinoma? J Urol (2005) 174(4 Pt 1):1218-21. doi: 10.1097/ 01.ju.0000173942.19990.40

3. Brookman-May SD, May M, Wolff I, Zigeuner R, Hutterer GC, Cindolo L, et al. Evaluation of the Prognostic Significance of Perirenal Fat Invasion and Tumor Size in Patients With pT1-pT3a Localized Renal Cell Carcinoma in a Comprehensive Multicenter Study of the CORONA Project. Can We Improve Prognostic Discrimination for Patients With Stage pT3a Tumors? Eur Urol (2015) 67(5):943-51. doi: 10.1016/j.eururo.2014.11.055

4. Poon SA, Gonzalez JR, Benson MC, McKiernan JM. Invasion of Renal Sinus Fat is Not an Independent Predictor of Survival in pT3a Renal Cell Carcinoma. BJU Int (2009) 103(12):1622-5. doi: 10.1111/j.1464-410X.2008.08239.x

5. Shah PH, Lyon TD, Lohse CM, Cheville JC, Leibovich BC, Boorjian SA, et al. Prognostic Evaluation of Perinephric Fat, Renal Sinus Fat, and Renal Vein Invasion for Patients With Pathological Stage T3a Clear-Cell Renal Cell Carcinoma. BJU Int (2019) 123(2):270-6. doi: 10.1111/bju.14523

6. Wang Z, Yu K, Zhu Y, Feng C, Liu C, Liu S, et al. Multiple Patterns of Perirenal Fat Invasion are Associated With a Poorer Prognosis Compared With Isolated Invasion: A Proposal for a Revision of T3aN0M0 Tnm Staging System. Front Oncol (2020) 10:336. doi: 10.3389/fonc.2020.00336

7. Oh JJ, Lee JK, Do Song B, Lee H, Lee S, Byun SS, et al. Accurate Risk Assessment of Patients With Pathologic T3an0m0 Renal Cell Carcinoma. Sci Rep (2018) 8(1):13914. doi: 10.1038/s41598-018-32362-w

8. Ravaud A, Motzer RJ, Pandha HS, George DJ, Pantuck AJ, Patel A, et al. Adjuvant Sunitinib in High-Risk Renal-Cell Carcinoma After Nephrectomy. N Engl J Med (2016) 375(23):2246-54. doi: 10.1056/NEJMoa1611406

9. Haas NB, Manola J, Dutcher JP, Flaherty KT, Uzzo RG, Atkins MB, et al. Adjuvant Treatment for High-Risk Clear Cell Renal Cancer: Updated Results including comprehensive combinations, may be useful for the further refinements of the TNM system.

\section{DATA AVAILABILITY STATEMENT}

The original contributions presented in the study are included in the article/supplementary material. Further inquiries can be directed to the corresponding authors.

\section{AUTHOR CONTRIBUTIONS}

PG and YW contributed to the design of the study. YH and DW were responsible for literature search. PG and JZ were responsible for data extraction and analysis. PG and ML were responsible for drafting the manuscript. YJ and YL approved the submitted version. All authors contributed to the article and approved the submitted version.

\section{FUNDING}

This study was supported by the Natural Science Foundation of Beijing Municipality (award no. 7192053). of a High-Risk Subset of the ASSURE Randomized Trial. JAMA Oncol (2017) 3(9):1249-52. doi: 10.1001/jamaoncol.2017.0076

10. Hayden JA, van der Windt DA, Cartwright JL, Cote P, Bombardier C. Assessing Bias in Studies of Prognostic Factors. Ann Intern Med (2013) 158 (4):280-6. doi: 10.7326/0003-4819-158-4-201302190-00009

11. Egger M, Davey Smith G, Schneider M, Minder C. Bias in Meta-Analysis Detected by a Simple, Graphical Test. BMJ (1997) 315(7109):629-34. doi: 10.1136/bmj.315.7109.629

12. Guyatt GH, Oxman AD, Vist GE, Kunz R, Falck-Ytter Y, Alonso-Coello P, et al. GRADE: An Emerging Consensus on Rating Quality of Evidence and Strength of Recommendations. BMJ (2008) 336(7650):924-6. doi: 10.1136/bmj.39489.470347.AD

13. Margulis V, Tamboli P, Matin SF, Meisner M, Swanson DA, Wood CG. Location of Extrarenal Tumor Extension Does Not Impact Survival of Patients With pT3a Renal Cell Carcinoma. J Urol (2007) 178(5):1878-82. doi: 10.1016/j.juro.2007.07.011

14. Bedke J, Buse S, Pritsch M, Macher-Goeppinger S, Schirmacher P, Haferkamp A, et al. Perinephric and Renal Sinus Fat Infiltration in pT3a Renal Cell Carcinoma: Possible Prognostic Differences. BJU Int (2009) 103(10):1349-54. doi: 10.1111/j.1464-410X.2008.08236.x

15. Bertini R, Roscigno M, Freschi M, Strada E, Petralia G, Pasta A, et al. Renal Sinus Fat Invasion in pT3a Clear Cell Renal Cell Carcinoma Affects Outcomes of Patients Without Nodal Involvement or Distant Metastases. J Urol (2009) 181(5):2027-32. doi: 10.1016/j.juro.2009.01.048

16. Kresowik TP, Johnson MT, Joudi FN. Combined Renal Sinus Fat and Perinephric Fat Renal Cell Carcinoma Invasion has a Worse Prognosis Than Either Alone. J Urol (2010) 184(1):48-52. doi: 10.1016/j.juro.2010.03.010

17. Park M, Shim M, Kim M, Song C, Kim CS, Ahn H. Prognostic Heterogeneity in T3aN0M0 Renal Cell Carcinoma According to the Site of Invasion. Urol Oncol (2017) 35(7):458.e417-58.e422. doi: 10.1016/j.urolonc.2016.05.019

18. Guo S, Liu Z, Li X, Yao K, Dong P, Chen D, et al. The Prognostic Value of the Site of Invasion in T3aN0M0 Clear Cell Renal Cell Carcinoma. Urol Oncol (2019) 37(5):301.e311-301.e317. doi: 10.1016/j.urolonc.2019.01.019

19. da Costa WH, Moniz RR, da Cunha IW, Fonseca FP, Guimaraes GC, de Cassio Zequi S. Impact of Renal Vein Invasion and Fat Invasion in pT3a Renal Cell Carcinoma. BJU Int (2012) 109(4):544-8. doi: 10.1111/j.1464-410X.2011.10366.x 
20. Baccos A, Brunocilla E, Schiavina R, Borghesi M, Rocca GC, Chessa F, et al. Differing Risk of Cancer Death Among Patients With Pathologic T3a Renal Cell Carcinoma: Identification of Risk Categories According to Fat Infiltration and Renal Vein Thrombosis. Clin Genitourin Cancer (2013) 11(4):451-7. doi: 10.1016/j.clgc.2013.05.006

21. Flood TA, Hogan K, Lavallee LT, Breau RH, Morash C, Belanger EC, et al. Evaluation of Individual and Cumulative Sites of Extrarenal Tumor Invasion in pT3a Clear Cell Renal Cell Carcinoma. Urol Oncol (2020) 38(2):42.e13-8. doi: 10.1016/j.urolonc.2019.09.030

22. Schiavina R, Borghesi M, Chessa F, Dababneh H, Bianchi L, Della Mora L, et al. The Prognostic Impact of Tumor Size on Cancer-Specific and Overall Survival Among Patients With Pathologic T3a Renal Cell Carcinoma. Clin Genitourin Cancer (2015) 13(4):e235-41. doi: 10.1016/j.clgc.2014.06.011

23. Peng D, He ZS, Li XS, Tang Q, Zhang L, Yang KW, et al. Partial Nephrectomy for T3aN0M0 Renal Cell Carcinoma: Shall We Step Forward? Int Braz J Urol (2017) 43(5):849-56. doi: 10.1590/S1677-5538.IBJU.2016.0598

24. Chen L, Ma X, Li H, Gu L, Li X, Gao Y, et al. Influence of Tumor Size on Oncological Outcomes of Pathological T3aN0M0 Renal Cell Carcinoma Treated by Radical Nephrectomy. PLoS One (2017) 12(3):e0173953. doi: 10.1371/journal.pone.0173953

25. Capitanio U, Stewart GD, Klatte T, Akdogan B, Roscigno M, Marszalek M, et al. Does the Unexpected Presence of Non-organ-confined Disease At Final Pathology Undermine Cancer Control in Patients With Clinical T1n0m0 Renal Cell Carcinoma Who Underwent Partial Nephrectomy? Eur Urol Focus (2018) 4(6):972-7. doi: 10.1016/j.euf.2017.02.020

26. Bailey GC, Boorjian SA, Ziegelmann MJ, Westerman ME, Lohse CM, Leibovich BC, et al. Urinary Collecting System Invasion is Associated With Poor Survival in Patients With Clear-Cell Renal Cell Carcinoma. BJU Int (2017) 119(4):585-90. doi: 10.1111/bju.13669

27. Garcia Marchinena P, Tobia I, Abreu D, de Cassio Zequi S, Jurado A, Gueglio G, et al. Prognostic Value of Perirenal and/or Sinus Fat Infiltration in Patients With pT3a Renal Cell Carcinoma: A Multicentre Cohort Study. LARCG Group. Actas Urol Esp (2019) 43(9):495-502. doi: 10.1016/j.acuro.2019.01.005

28. Lai GS, Li JR, Wang SS, Chen CS, Yang CK, Hung SC, et al. Prognostic Evaluation of the Site of Invasion in Pathological Stage T3a Renal Cell Carcinoma. In Vivo (2021) 35(2):1083-9. doi: 10.21873/invivo.12353

29. Verhoest G, Avakian R, Bensalah K, Thuret R, Ficarra V, Artibani W, et al. Urinary Collecting System Invasion is an Independent Prognostic Factor of Organ Confined Renal Cell Carcinoma. J Urol (2009) 182(3):854-9. doi: 10.1016/j.juro.2009.05.017

30. Anderson CB, Clark PE, Morgan TM, Stratton KL, Herrell SD, Davis R, et al. Urinary Collecting System Invasion is a Predictor for Overall and DiseaseSpecific Survival in Locally Invasive Renal Cell Carcinoma. Urology (2011) 78 (1):99-104. doi: 10.1016/j.urology.2011.02.039

31. Chen L, Ma X, Li H, Li X, Gao Y, Zhang Y, et al. Invasion of the Urinary Collecting System is an Independent Prognostic Factor in pT3 Renal Cell Carcinoma. Urol Oncol (2016) 34(7):293 e211-296. doi: 10.1016/j.urolonc.2016.02.019

32. Palapattu GS, Pantuck AJ, Dorey F, Said JW, Figlin RA, Belldegrun AS. Collecting System Invasion in Renal Cell Carcinoma: Impact on Prognosis and Future Staging Strategies. J Urol (2003) 170(3):768-72; discussion 772. doi: 10.1097/01.ju.0000082580.13597.a2

33. Srivastava A, Patel HD, Joice GA, Semeriian A, Gorin MA, Johnson MH, et al. Incidence of T3a Up-Staging and Survival After Partial Nephrectomy: Sizestratified Rates and Implications for Prognosis. Urol Oncol (2018) 36(1):12 e17-.e13. doi: 10.1016/j.urolonc.2017.09.005

34. Shvero A, Nativ O, Abu-Ghanem Y, Zilberman D, Zaher B, Levitt M, et al. Oncologic Outcomes of Partial Nephrectomy for Stage T3a Renal Cell Cancer. Clin Genitourin Cancer (2018) 16(3):e613-7. doi: 10.1016/j.clgc.2017.10.016

35. Murphy AM, Gilbert SM, Katz AE, Goluboff ET, Sawczuk IS, Olsson CA, et al. ReEvaluation of the Tumour-Node-Metastasis Staging of Locally Advanced Renal Cortical Tumours: Absolute Size (T2) is More Significant Than Renal Capsular Invasion (T3a). BJU Int (2005) 95(1):27-30. doi: 10.1111/j.1464-410X.2005.05244.x

36. Kirkali Z, Algaba F, Scarpelli M, Trias I, Selvaggi FP, Van Poppel H. What Does the Urologist Expect From the Pathologist (and What can the Pathologists Give) in Reporting on Adult Kidney Tumour Specimens? Eur Urol (2007) 51(5):1194-201. doi: 10.1016/j.eururo.2006.11.024

37. Grignon D, Paner GP. Renal Cell Carcinoma and the Renal Sinus. Adv Anat Pathol (2007) 14(2):63-8. doi: 10.1097/PAP.0b013e318032452e
38. Wood EL, Adibi M, Qiao W, Brandt J, Zhang M, Tamboli P, et al. Local Tumor Bed Recurrence Following Partial Nephrectomy in Patients With Small Renal Masses. J Urol (2018) 199(2):393-400. doi: 10.1016/j.juro.2017.09.072

39. Shah PH, Moreira DM, Okhunov Z, Patel VR, Chopra S, Razmaria AA, et al. Positive Surgical Margins Increase Risk of Recurrence After Partial Nephrectomy for High Risk Renal Tumors. J Urol (2016) 196(2):327-34. doi: 10.1016/j.juro.2016.02.075

40. Shah PH, Moreira DM, Patel VR, Gaunay G, George AK, Alom M, et al. Partial Nephrectomy is Associated With Higher Risk of Relapse Compared With Radical Nephrectomy for Clinical Stage T1 Renal Cell Carcinoma Pathologically Up Staged to T3a. J Urol (2017) 198(2):289-96. doi: 10.1016/j.juro.2017.03.012

41. Bensalah K, Pantuck AJ, Rioux-Leclercq N, Thuret R, Montorsi F, Karakiewicz PI, et al. Positive Surgical Margin Appears to Have Negligible Impact on Survival of Renal Cell Carcinomas Treated by Nephron-Sparing Surgery. Eur Urol (2010) 57(3):466-71. doi: 10.1016/j.eururo.2009.03.048

42. Tabayoyong W, Abouassaly R, Kiechle JE, Cherullo EE, Meropol NJ, Shah ND, et al. Variation in Surgical Margin Status by Surgical Approach Among Patients Undergoing Partial Nephrectomy for Small Renal Masses. J Urol (2015) 194(6):1548-53. doi: 10.1016/j.juro.2015.06.076

43. Tellini R, Antonelli A, Tardanico R, Fisogni S, Veccia A, Furlan MC, et al. Positive Surgical Margins Predict Progression-Free Survival After Nephronsparing Surgery for Renal Cell Carcinoma: Results From a Single Center Cohort of 459 Cases With a Minimum Follow-Up of 5 Years. Clin Genitourin Cancer (2019) 17(1):e26-31. doi: 10.1016/j.clgc.2018.08.004

44. Shimizu T, Miyake M, Hori S, Iida K, Ichikawa K, Sakamoto K, et al. Clinical Significance of Tumor Size, Pathological Invasion Sites Including Urinary Collecting System and Clinically Detected Renal Vein Thrombus as Predictors for Recurrence in Pt3a Localized Renal Cell Carcinoma. Diagn (Basel) (2020) 10(3):154-66. doi: 10.3390/diagnostics10030154

45. Chevinsky M, Imnadze M, Sankin A, Winer A, Mano R, Jakubowski C, et al. Pathological Stage T3a Significantly Increases Disease Recurrence Across All Tumor Sizes in Renal Cell Carcinoma. J Urol (2015) 194(2):310-5. doi: 10.1016/j.juro.2015.02.013

46. Chen K, Lee BL, Huang HH, Tan BY, Lee LS, Ng LG, et al. Tumor Size and Fuhrman Grade Further Enhance the Prognostic Impact of Perinephric Fat Invasion and Renal Vein Extension in T3a Staging of Renal Cell Carcinoma. Int J Urol (2017) 24(1):51-8. doi: 10.1111/iju.13237

47. Lam JS, Klatte T, Patard JJ, Goel RH, Guille F, Lobel B, et al. Prognostic Relevance of Tumour Size in T3a Renal Cell Carcinoma: A Multicentre Experience. Eur Urol (2007) 52(1):155-62. doi: 10.1016/j.eururo.2007.01.106

48. Siddiqui SA, Frank I, Leibovich BC, Cheville JC, Lohse CM, Zincke H, et al. Impact of Tumor Size on the Predictive Ability of the pT3a Primary Tumor Classification for Renal Cell Carcinoma. J Urol (2007) 177(1):59-62. doi: 10.1016/j.juro.2006.08.069

49. Yoo C, Song C, Hong JH, Kim CS, Ahn H. Prognostic Significance of Perinephric Fat Infiltration and Tumor Size in Renal Cell Carcinoma. J Urol (2008) 180(2):486-91; discussion 491. doi: 10.1016/j.juro.2008.04.034

50. Dabestani S, Beisland C, Stewart GD, Bensalah K, Gudmundsson E, Lam TB, et al. Increased Use of Cross-Sectional Imaging for Follow-Up Does Not Improve Post-Recurrence Survival of Surgically Treated Initially Localized R.C.C.: Results From a European Multicenter Database (R.E.C.U.R.). Scand J Urol (2019) 53(1):14-20. doi: 10.1080/21681805.2019.1588919

51. Lenis AT, Donin NM, Johnson DC, Faiena I, Salmasi A, Drakaki A, et al. Adjuvant Therapy for High Risk Localized Kidney Cancer: Emerging Evidence and Future Clinical Trials. J Urol (2018) 199(1):43-52. doi: 10.1016/j.juro.2017.04.092

52. Gross-Goupil M, Kwon TG, Eto M, Ye D, Miyake H, Seo SI, et al. Axitinib Versus Placebo as an Adjuvant Treatment of Renal Cell Carcinoma: Results From the Phase III, Randomized ATLAS Trial. Ann Oncol (2018) 29 (12):2371-8. doi: 10.1093/annonc/mdy454

53. Eisen T, Frangou E, Oza B, Ritchie AWS, Smith B, Kaplan R, et al. Adjuvant Sorafenib for Renal Cell Carcinoma At Intermediate or High Risk of Relapse: Results From the SORCE Randomized Phase Iii Intergroup Trial. J Clin Oncol (2020) 38(34):4064-75. doi: 10.1200/JCO.20.01800

54. Massari F, Di Nunno V, Mollica V, Graham J, Gatto L, Heng D. Adjuvant Tyrosine Kinase Inhibitors in Treatment of Renal Cell Carcinoma: A MetaAnalysis of Available Clinical Trials. Clin Genitourin Cancer (2019) 17(2): e339-44. doi: 10.1016/j.clgc.2018.12.011

55. Gu L, Li H, Wang Z, Wang B, Huang Q, Lyu X, et al. A Systematic Review and Meta-Analysis of Clinicopathologic Factors Linked to Oncologic Outcomes for 
Renal Cell Carcinoma With Tumor Thrombus Treated by Radical Nephrectomy With Thrombectomy. Cancer Treat Rev (2018) 69:112-20. doi: 10.1016/ j.ctrv.2018.06.014

Conflict of Interest: The authors declare that the research was conducted in the absence of any commercial or financial relationships that could be construed as a potential conflict of interest.
Copyright (C) 2021 Guo, Wang, Han, Wei, Zhao, Li, Jiang and Luo. This is an openaccess article distributed under the terms of the Creative Commons Attribution License (CC BY). The use, distribution or reproduction in other forums is permitted, provided the original author(s) and the copyright owner(s) are credited and that the original publication in this journal is cited, in accordance with accepted academic practice. No use, distribution or reproduction is permitted which does not comply with these terms. 